\title{
Ultrasonography of the spleen. Pictorial essay.
}

\author{
Liliana Chiorean ${ }^{1}$, Mihnea Zdrenghea ${ }^{2}$, Radu Badea ${ }^{3}$
}

${ }^{1}$ Department of Radiology and Computed Tomography, "Octavian Fodor" Institute of Gastroenterology and Hepatology, ${ }^{2}$ Hematology Department, Institute of Oncology, ${ }^{3}$ Department of Ultrasonography, “Octavian Fodor" Institute of Gastroenterology and Hepatology, "Iuliu Haţieganu" University of Medicine and Pharmacy, Cluj-Napoca, Romania

\section{Abstract}

Contrast-enhanced ultrasound is widely indicated in the study of splenic diseases, especially due to its good specificity in the differentiation of benign from malignant splenic lesions. The purpose of this pictorial essay is to offer a review of the most common splenic pathologies, while illustrating them with sonographic images.

Keywords: spleen, ultrasonography, contrast-enhanced ultrasound, colour-Doppler, elastography

\section{Introduction}

Ultrasonography (US) is extensively used in imaging the abdomen, being frequently the first method of choice for an abdominal survey. As part of the abdominal US examination, US of the spleen still plays an important role in diagnosis, even though other imagistic modalities are becoming increasingly competitive [1].

Even if it has been named 'the forgotten organ', careful examination of the spleen can bring important diagnostic clues in pathologies such as oncologic, hematologic, infectious, and metabolic, abdominal trauma, portal hypertension, and many other focal or diffuse splenic changes of different etiologies.

\section{Examination Technique}

The spleen should be examined with the patient on their back or right side, using a 3-5 MHz curved linear transducer

Received 14.12.2013 Accepted 30.12.2013

Med Ultrason

2014, Vol. 16, No 1, 48-59

Corresponding author: Radu Badea,

Institute of Gastroenterology and Hepatology,

Department of Ultrasound

"Iuliu Hatieganu" University of Medicine

and Pharmacy Cluj Napoca,

19-21 Croitorilor Str, 400163, Cluj Napoca, RO

Email: rbadea@umfcluj.ro while the patient exhales in order to avoid spleen coverage by the left lung tissue. The best window to view the spleen is at the level of $10^{\text {th }}$ or $11^{\text {th }}$ intercostal spaces, on the left midaxillary line. Colour Doppler imaging may sometimes assist in an accurate diagnosis, but it can only assess the macrocirculation. Contrast-enhanced US (CEUS) can assess both macro- and microcirculation in real-time. It requires a skilled user, being highly operator dependent. The examination should be made using a high-frequency transducer, and tissue harmonic imaging in the presence of a low mechanical index (MI) [1-3]. In our institution, we usually inject intravenously $2.4 \mathrm{ml}$ of contrast-agent SonoVue (Bracco, Italy), followed by $10 \mathrm{cc}$ of saline solution. Injection of this contrast agent can be immediately repeated, even if rarely necessary. Elastography is a new investigation technique used to assess the elasticity of tissues, being the imagistic equivalent of palpation used from ancient times in medicine. The technique is different for every US machine producer with software for elastography available [4].

\section{Normal Appearance}

On US, the spleen is crescent shaped, with a smooth outer convexity, and an inner margin intended or nodulous [1]. Its normal size is less than $12 \mathrm{~cm}$ in length, and it may decrease with age [5]. The normal appearance of the parenchyma is very homogeneous and uniform, with an echogenicity slightly greater than that of normal he- 
patic parenchyma [6]. At CEUS, the phases of contrast enhancement are broadly divided into arterial (10-25 s), portal venous (30-120 s), and late phases (over $120 \mathrm{~s}$ ) [7]. During the arterial time, the arterial splenic vessels are displayed (fig 1a), while in the portal venous phase, the splenic parenchyma becomes homogenously enhanced (also called the parenchymal phase) (fig 1b).

\section{Abnormal Findings}

Focal lesions in the spleen are rare in comparison with those located in other solid viscera. The accurate analysis of internal architecture of the lesion can give us important diagnostic clues, due to the US's ability in depicting the calcifications (long term process) or gas within a lesion (usually originating from bacterial infection), those being most frequently related to benign findings. The malignant lesions are more frequently multifocal (especially metastasis), and tend to be diffuse and ill-defined due to rapid growth [8]. Because the US appearance of disease in the spleen is rather nonspecific, a thorough knowledge of the patient's history and associated symptoms may be very helpful in the differential diagnosis [6].

Different patterns of enhancement for focal splenic lesions have been described at CEUS examination: benign lesions that have constantly nonenhancing or isoenhancing pattern and malignant lesions with enhancement in arterial time, followed by rapid wash-out, or progressively hypoenhacing with heterogenous aspect. This last aspect was described in some studies as having $100 \%$ sensitivity, and $83 \%$ specificity for malignancy $[2,3,9-12]$.

\section{Diffuse Splenic Disease}

The basic finding in many diseases of the spleen is the acute or chronic increase in the size of the organ. Splenomegaly can be caused by infections, liver or blood diseases, problems with the lymph system, or other conditions [5].
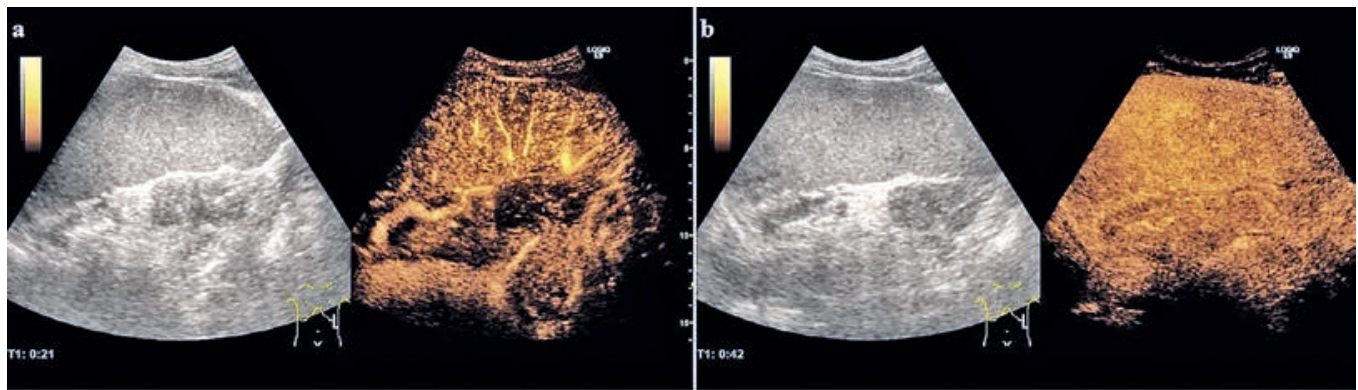

Fig 1. Gray-scale and CEUS of the spleen (dual examination): a) arterial time (at 21 seconds after contrast agent administration) - the arterial splenic vessels are seen; b) portal time (42 seconds), the splenic parenchyma becomes homogenously enhanced (also called the parenchymal time).
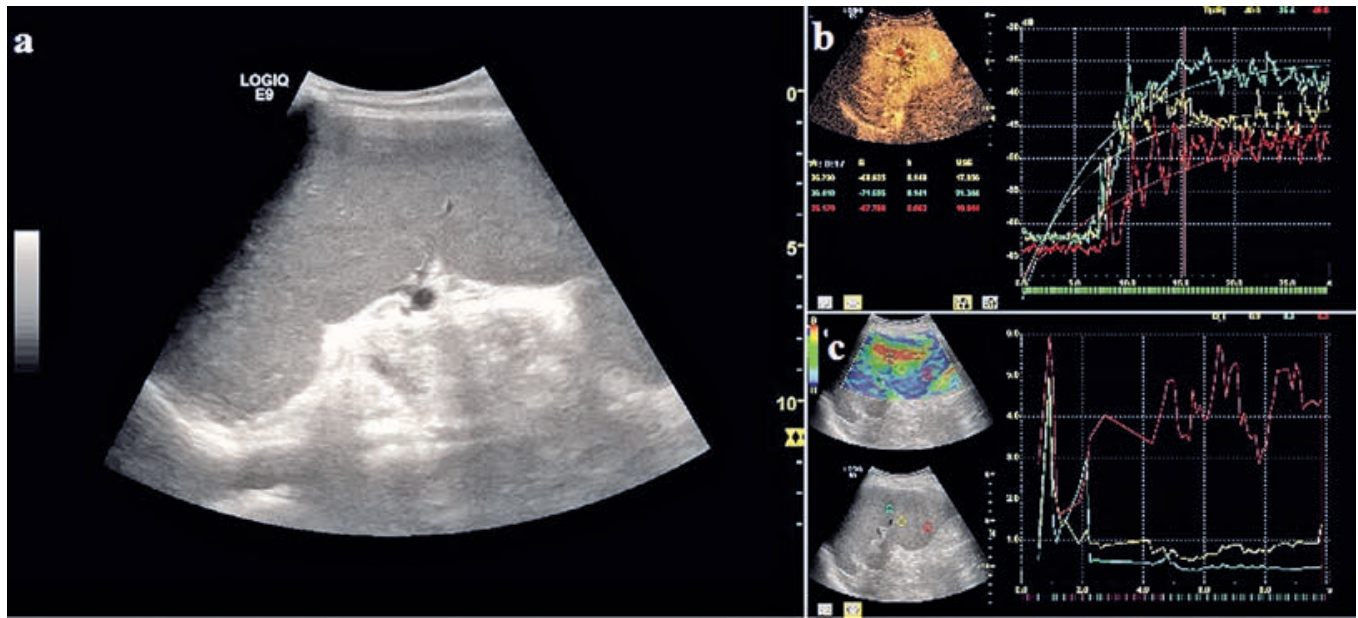

Fig 2. Splenomegaly. a) 2-D ultrasound - enlarged spleen; b) CEUS - inhommogenous enhancement during the arterial time, starting at 7 seconds after contrast administration, and reaching the peack at 17 seconds; c) strain elastogram, showing inhommogenous pattern, stiffer subcapsulary. 
Presence of an enlarged spleen may need fusion of multiple images or the use of panoramic images in order to view the entire organ (fig 2a). The aspect of enhancement in CEUS is nonspecific and inconstant (fig 2b). In marked splenomegaly, a delay in contrast up-taken and a less intense opacification of splenic parenchyma may be found [2]. The elastogram may present with an inhomogeneous pattern, with stiffer areas located subcapsular, due to enlarged parenchyma compressed under the splenic capsule (fig 2c).

\section{Focal Splenic Lesions}

Cysts

Cysts are the most common benign lesions of the spleen, and usually present no diagnostic problems. They may be primary or acquired. The primary ones are true cysts, with epithelium, endothelium, and membrane lining, and may be congenital, parasitic, or neoplastic. Most often primary cysts are simple, uniloculated, but they may also present incomplete septations, or punctate cal-

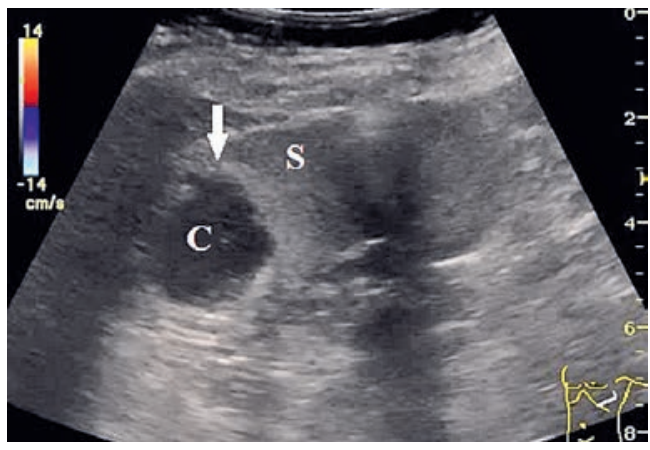

Fig 3. Hydatic cyst. Round, anechoic splenic lesion (arrow), presenting acoustic enhancement, and no vascular signal at Doppler examination $(\mathrm{C}=$ cyst, $\mathrm{S}=$ spleen $)$. cifications. The secondary cysts, 'false', have no cellular lining, and may be the result of a trauma, a degenerative evolution of a splenic infarction, or a post-necrotic evolution of an abscess. In these cases, due to bleeding or necrosis, the result is a mixed echographic pattern, partially cystic. The differential diagnosis may be difficult, and a puncture may be needed (fig 3, fig 4) [8,13-15].

\section{Infarction}

Infarct usually appears as triangular hypoechoic areas, with the base on the splenic capsule and pointing towards the hilum, without vascular signal inside. The margins of the lesion can be irregular, ill-defined (especially in the acute stage), or well delineated, and the texture may be homogenous or inhomogeneous. On CEUS, it is immediately recognized due to total absence of enhancement. The interrupted artery may be visible in the arterial time. It has a high tendency to spontaneous healing (fig 5-7) $[2,10,13]$.

Abscess

Splenic abscess is rare and represents a collection of pus commonly caused by hematogenous spread of infection. Micro-abscesses in patients with bacterial endocarditis, or infectious complications of hematomas or infarctions may also be encountered [6]. They have various and nonspecific sonographic appearances, depending on their etiology and size. Large abscesses are often hypoechoic, with a thick irregular wall [16]. They show no uptake of contrast agent at CEUS, but may present a hyperechoic, enhancing rim. Enhancing septa may be seen, but no sign of contrast microcirculation is seen within the internal fluid, debris, and necrotic components (fig 8) $[10,17,18]$. Due to the need of rapid diagnosis and early treatement, percutaneous fine-needle aspiration guided by sonography is required as soon as the
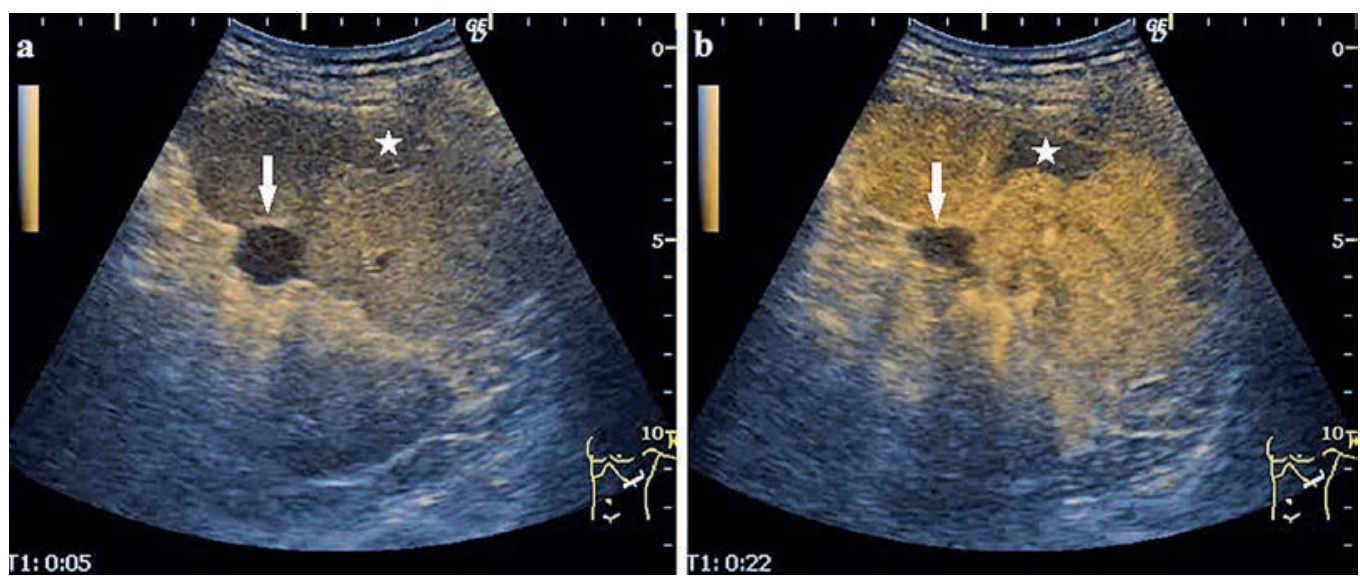

Fig 4. Cystic lesion located at the level of splenic hilum (arrows), CEUS examination. a) aspect at 5 seconds after contrast administration - the anechoic lesion can be well seen; b) arterial time - the cystic lesion presents no enhancement, and another unenhanced triangular shaped area can be seen (asterisk), representing a secondary splenic infarctus, due to compression of hilar splenic vessels, produced by the cystic tumor located at this level. 


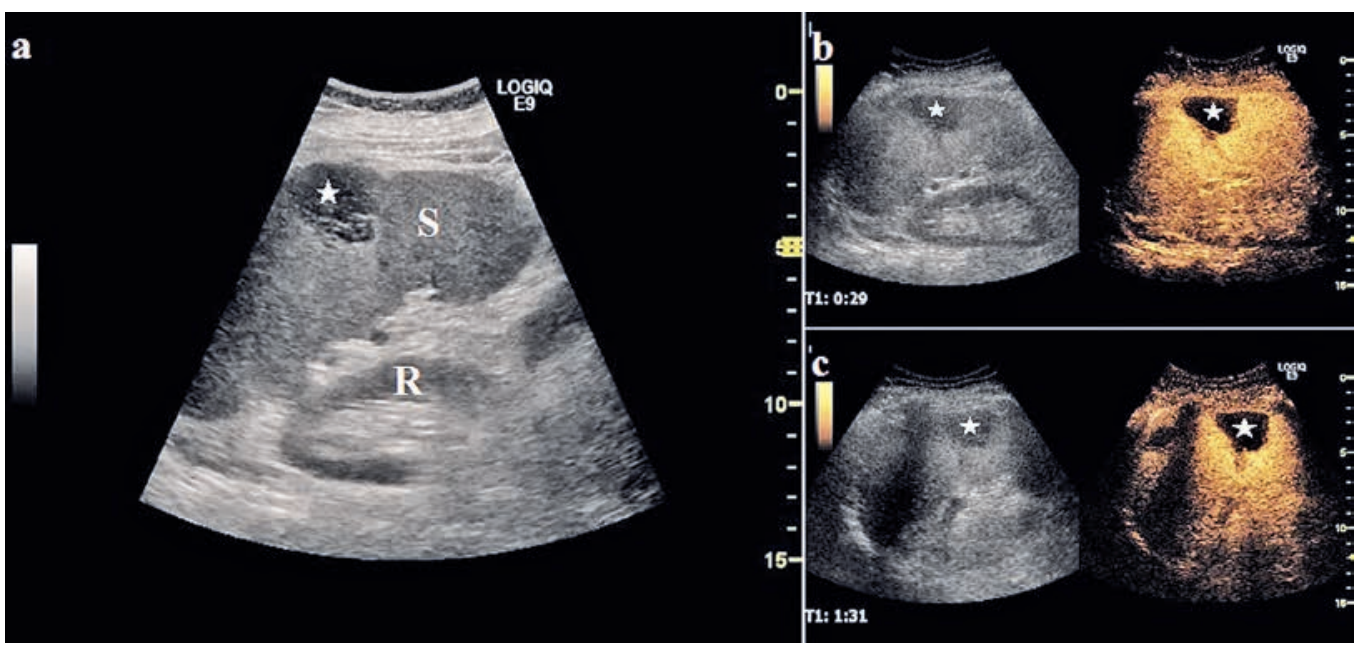

Fig 5. Splenic infarct. a) sonogram shows a triangular hypoechoic lesion (asterisk); b) CEUS, arterial time - the splenic lesion is unenhanced; c) CEUS, prenchymal phase - the lesion presents no enhancement ( $\mathrm{S}=$ spleen; $\mathrm{R}=$ left kidney).
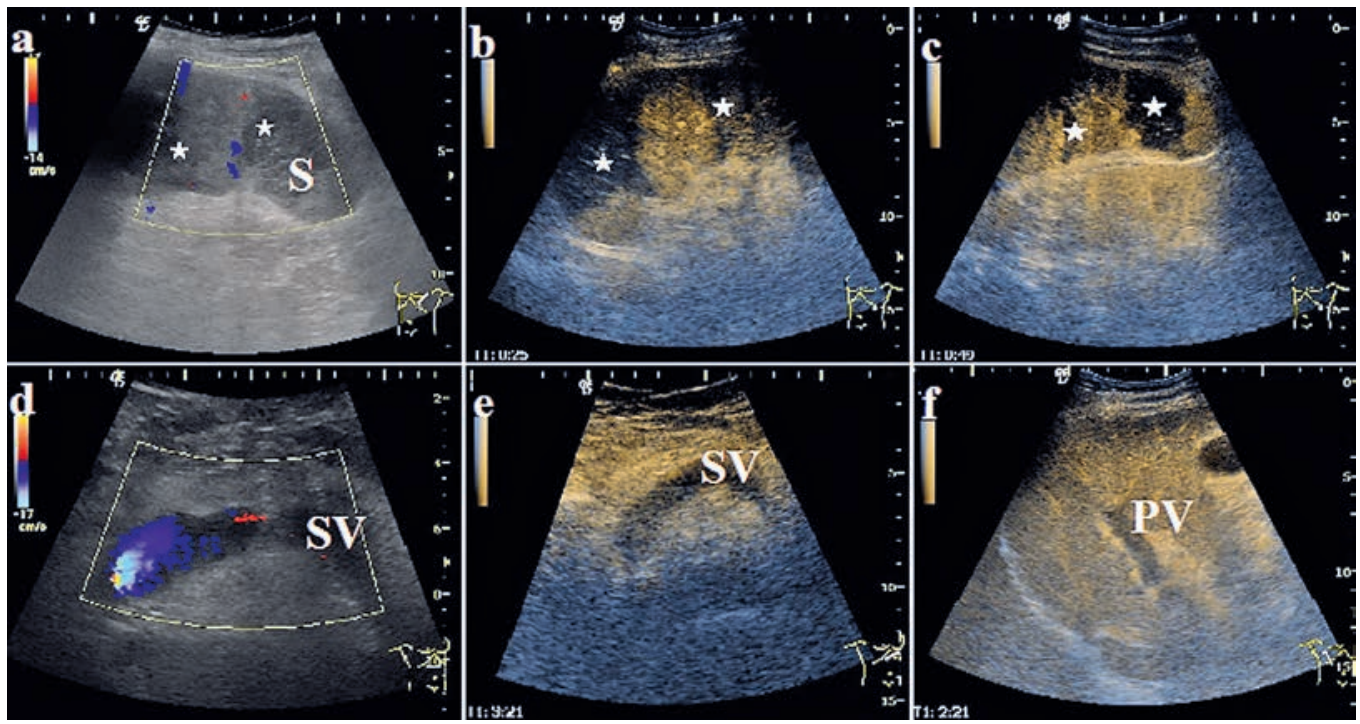

Fig 6. Splenic infarcts (asterisk) in a patient with splenic and portal vein thrombosis. a) Doppler examination - showing the absence of the vascular signal inside the hypoechoic, wedge-shaped triangular lesions; b,c) CEUS of the spleen - the lessions shows no contrast agent uptake, nor in the arterial, neither in the parenchymal phase; d) Doppler examinantion showing the absence of the vascular signal at the level of the splenic vein; e) CEUS reveals absence of contrast agent inside the splenic vein in the late venous phase; f) CEUS late phase - no enhancement inside the portal vein $(S=$ spleen, $S V=$ splenic vein, $\mathrm{PV}=$ portal vein $)$.

suspicion has been raised, especially because the clinical diagnostic triad is present in only $44 \%$ of cases $[13,19]$.

\section{Hemangiomas}

Hemangioma is the most common benign solid lesion of the spleen characterized by a proliferation of blood-filled spaces lined and separated by the endothelium. It is usually a solitary, well-marginated lesion, having less than $2 \mathrm{~cm}$ in size. The sonographical appearance is different for the two histological types of hemangiomas: the cavernous hemangioma appears as a mixed echogenic or hypoechoic structure, with possible calcifications or cystic component; a capillary hemangioma is hyperechoic, with well-defined margins. The most frequent complications are rupture and bleeding $[1,6,13]$. At CEUS, capillary hemangiomas are usually isoenhancing with the ad- 


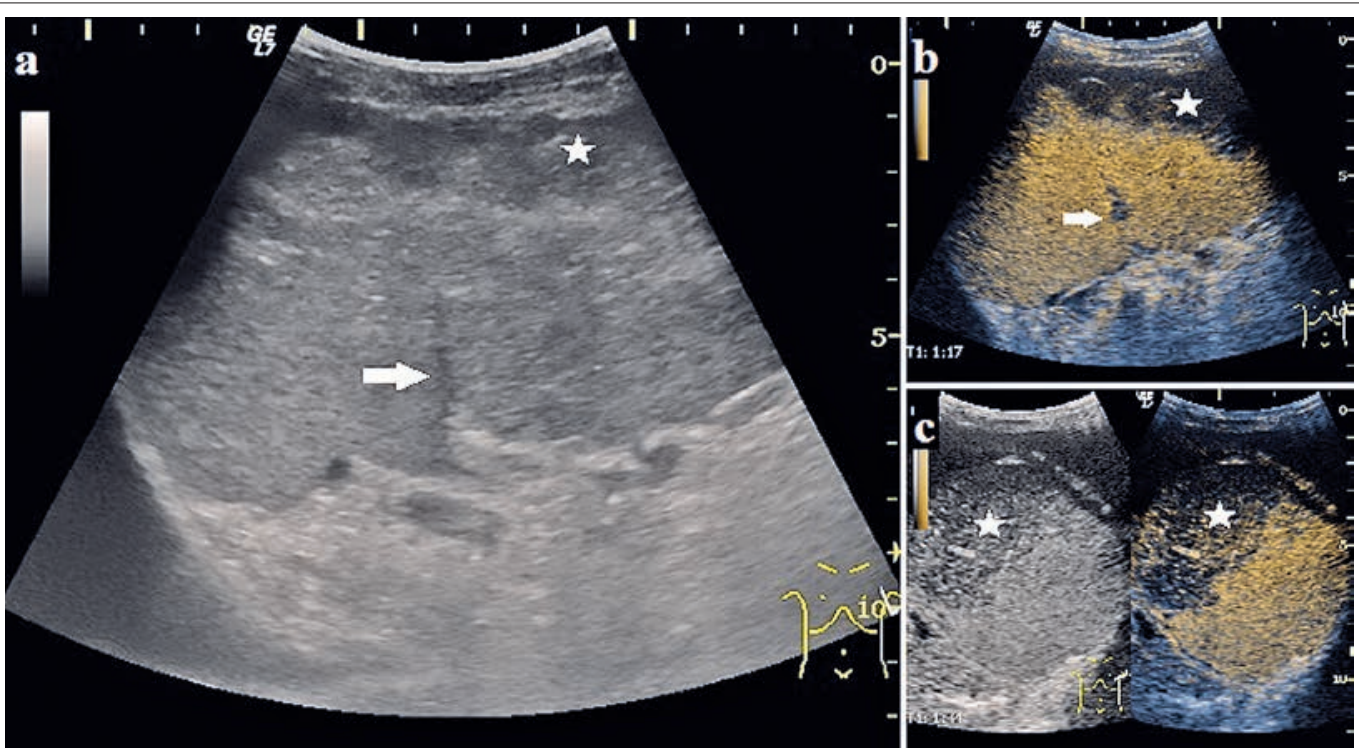

Fig 7. Splenic infarcts through microembolisations. a) Sonogram showing an inhomogeneous spleen, with a large hypoechoic area, wedge-shaped (asterisk), and others smaller (arrow); b,c) no enhancement inside the splenic lesions.
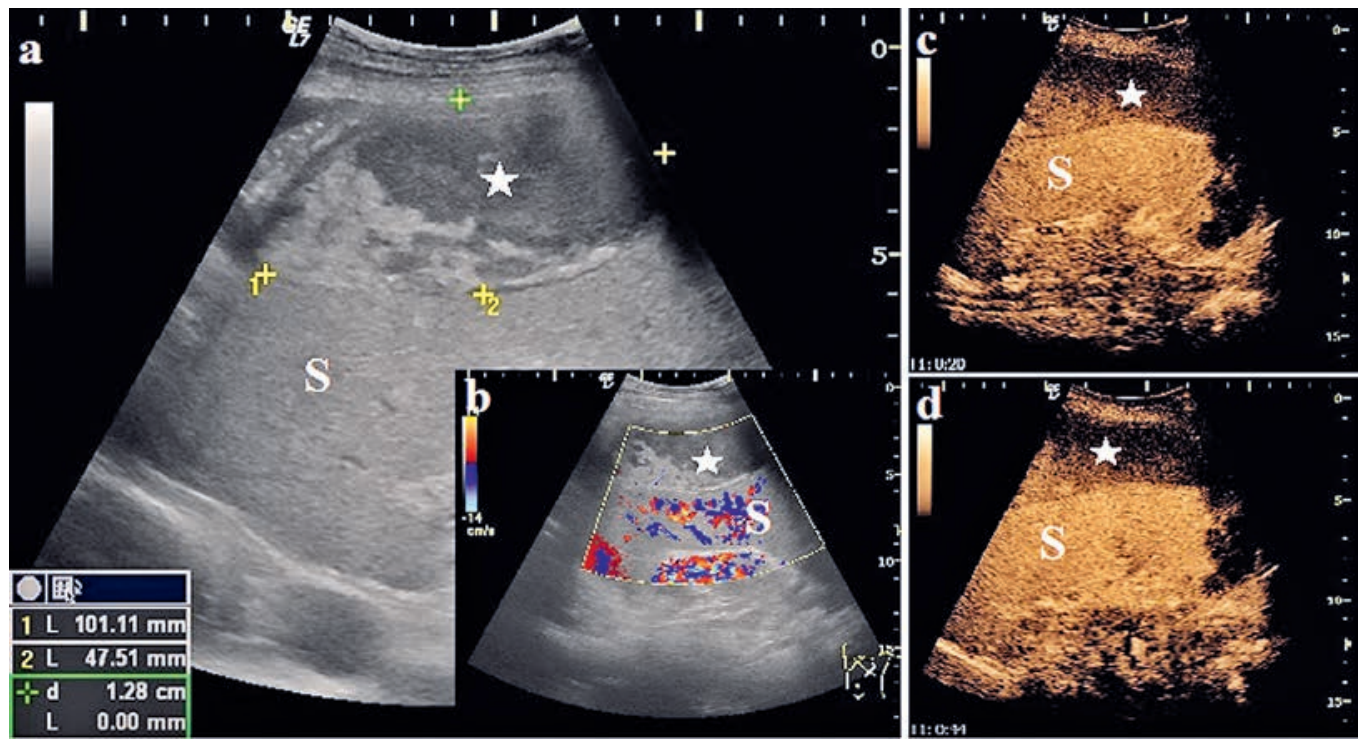

Fig 8. Splenic abscess. a) Sonogram shows a large, hypoechoic splenic lesion, having inhomogenous content, with debris (asterisk); b) absence of vascular signal inside the lesion; c,d) arterial and portal time of CEUS examination - the splenic lesion is unenhanced $(\mathrm{S}=$ spleen $)$.

jacent splenic parenchyma. A hyperechoic splenic lesion on gray-scale, undetectable after contrast-agent administration is diagnosed as a hemangioma [10]. Cavernous hemangiomas can present a diffuse or centripetal filling-in, rapid or slow, but with a greater enhancement degree (fig 9, fig 10) [2].

\section{Trauma}

Computed-tomography is the reference standard in detecting and monitoring splenic trauma [1]. Fissures may be hyperechoic or hypoechoic compared to splenic parenchyma, contusions appear as slightly hypoechoic, ill-defined areas, and lacerations may be seen as clearly hypoechoic band, linear or branched, perpendicular to the splenic surface $[2,20]$. A fresh hematoma imposes a hyperechoic structural change within the splenic parenchyma, while an organized hematoma shows a varied echogenic structure, and a decreased or a lack of con- 


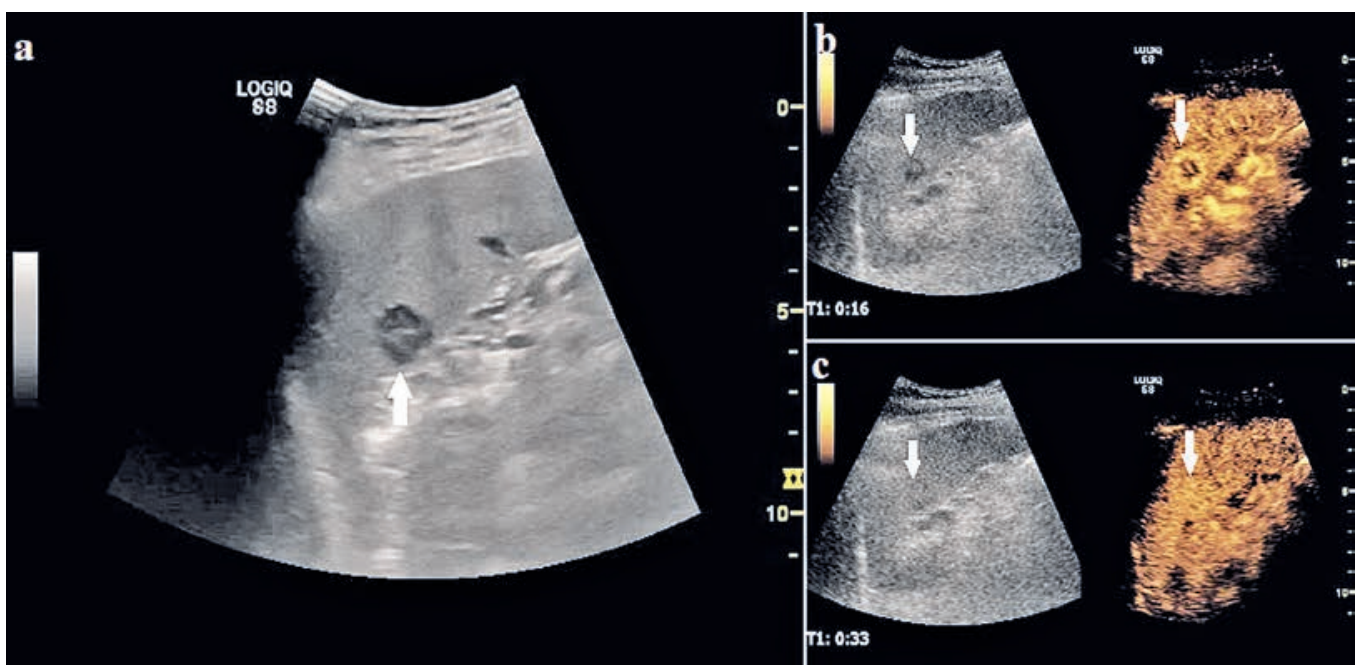

Fig 9. Splenic hemangioma (asterisk). a) Splenic sonography shows a hypoechoic splenic lesion in an asymptomatic patient; $b, c)$ the splenic lesion is isoenhancing comparing to the adjacent splenic parenchyma.
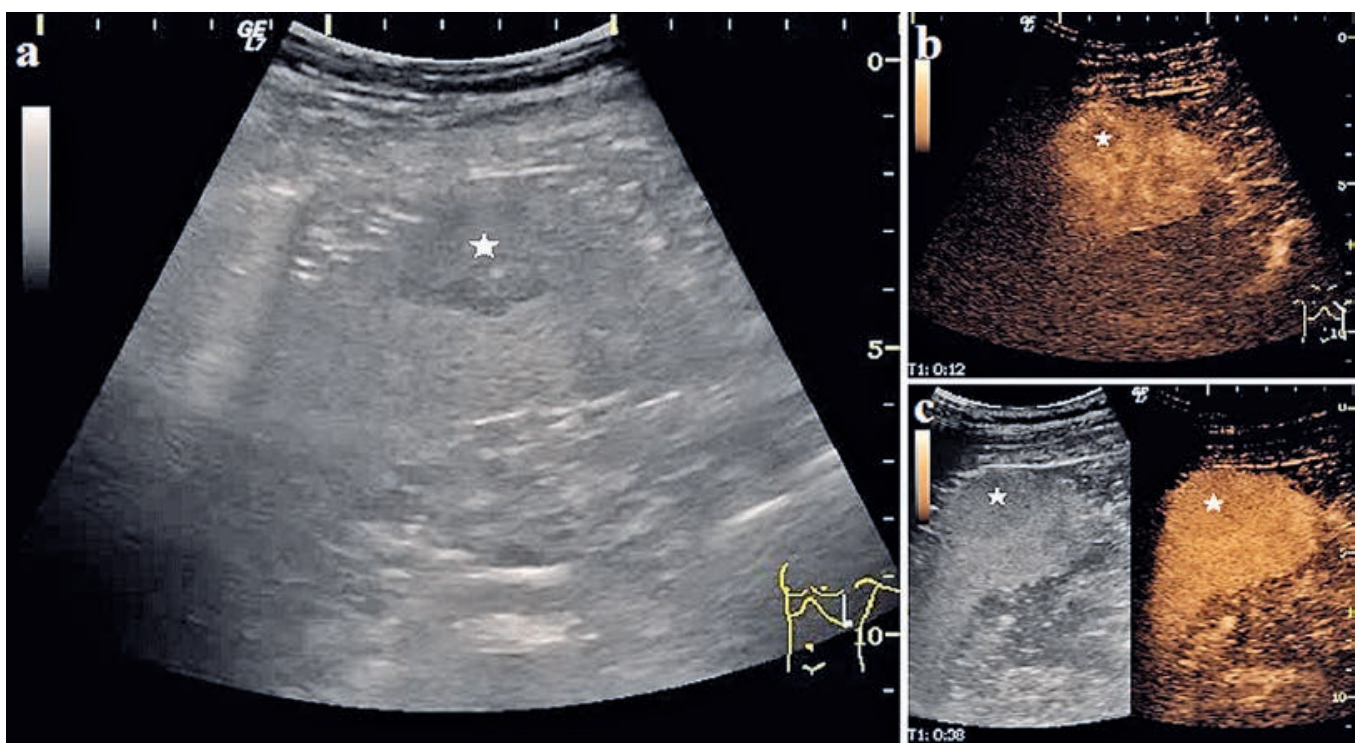

Fig 10. Splenic hemangioma (arrows). a) 2-D splenic US reveals a round, hypoechoic, well-delineated splenic lesion; b) the lesion present a centripetal fill-in pattern of enhancement; c) during the portal, parenchymal phase, the lesion could not be distinguished, being isoenhanced with the rest of splenic parenchyma.

trast agent uptake at CEUS, better evident during the late phase of enhancement $[1,21]$.

More severe lesions can also cause a subcapsular hematoma. Free fluid in the perisplenic region of the abdominal cavity is an indirect sign of a ruptured spleen [22].

Color Doppler and CEUS are helpful in detecting hemorrhage and posttraumatic splenic pseudoaneurysms.
With CEUS the acute hemorrhages and pseudoaneurysms are indicated by an early-phase hyperechoic pool or jet within the splenic parenchyma or perisplenic hematomas (fig 11-14) [21].

\section{Lymphoma}

Lymphoma is one of the most common primary malignancy of the spleen, being more common than splenic metastases. The spleen usually is larger, but a normal 

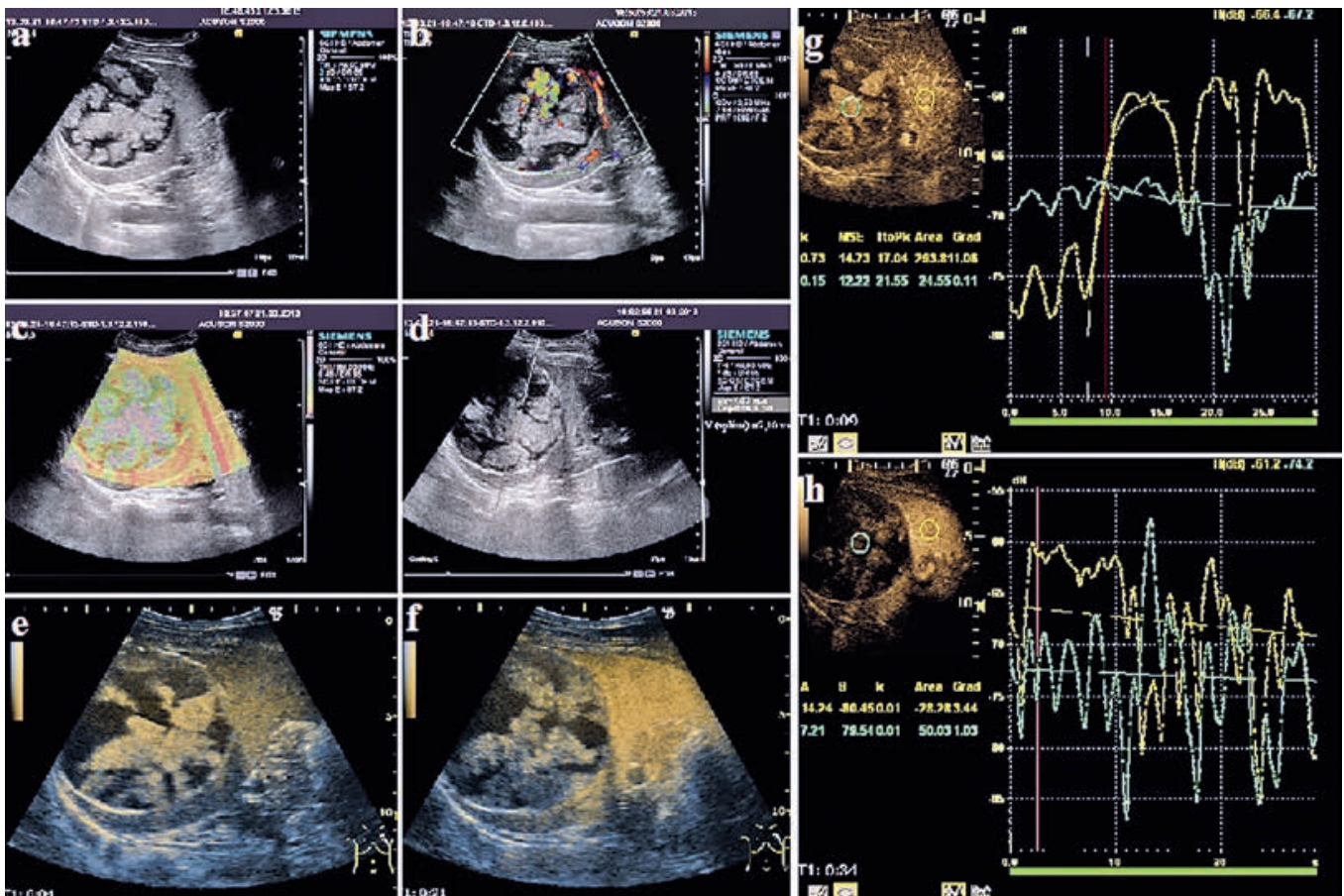

Fig 11. Intraparenchymal splenic hematoma in a young patient who recently suffered an abdominal trauma. a) 2-D US - subcapsular splenic mass, well delineated, with mixed content, partially liquid, partially solid; b) Doppler-US - partial vascular signal inside the lesion, possibly representing active bleeding; c) Strainelastography - the splenic focal lesion is less stiff compared to the adjacent splenic parenchyma; d) ARFI - velocities inside the lesion up till $1.07 \mathrm{~m} / \mathrm{sec}$, confirming the strain result of a soft content; e,f) CEUS reevaluation after 4 days - there's no contrast agent inside the lesion, which sustains the absence of an active bleeding at the time the examination was performed; $g, h$ ) contrast-curves - no enhancement inside the splenic lesion, and normal contrast-agent uptake of the adjacent parenchyma.

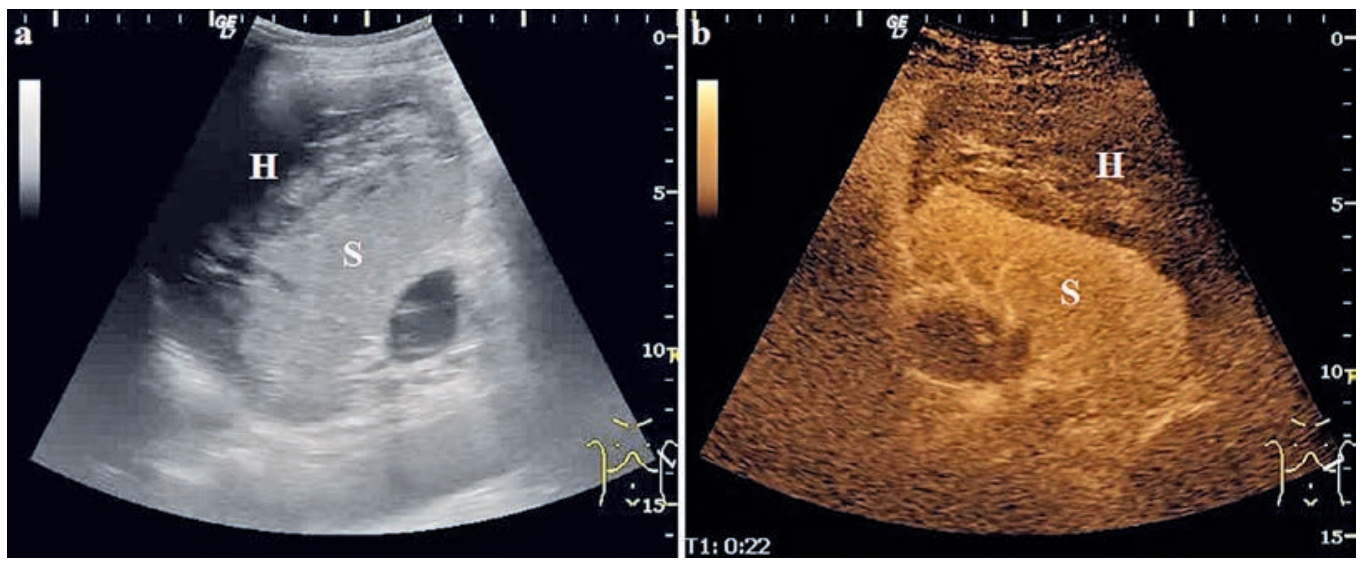

Fig 12. Subcapsular splenic hematoma. a) peripheral crescent-shaped hematoma; b) no contrast-agent extravasation inside the hematoma was seen, which represents absence of active bleeding at the time the examination has been performed $(\mathrm{S}=$ spleen, $\mathrm{H}=$ hematoma). 

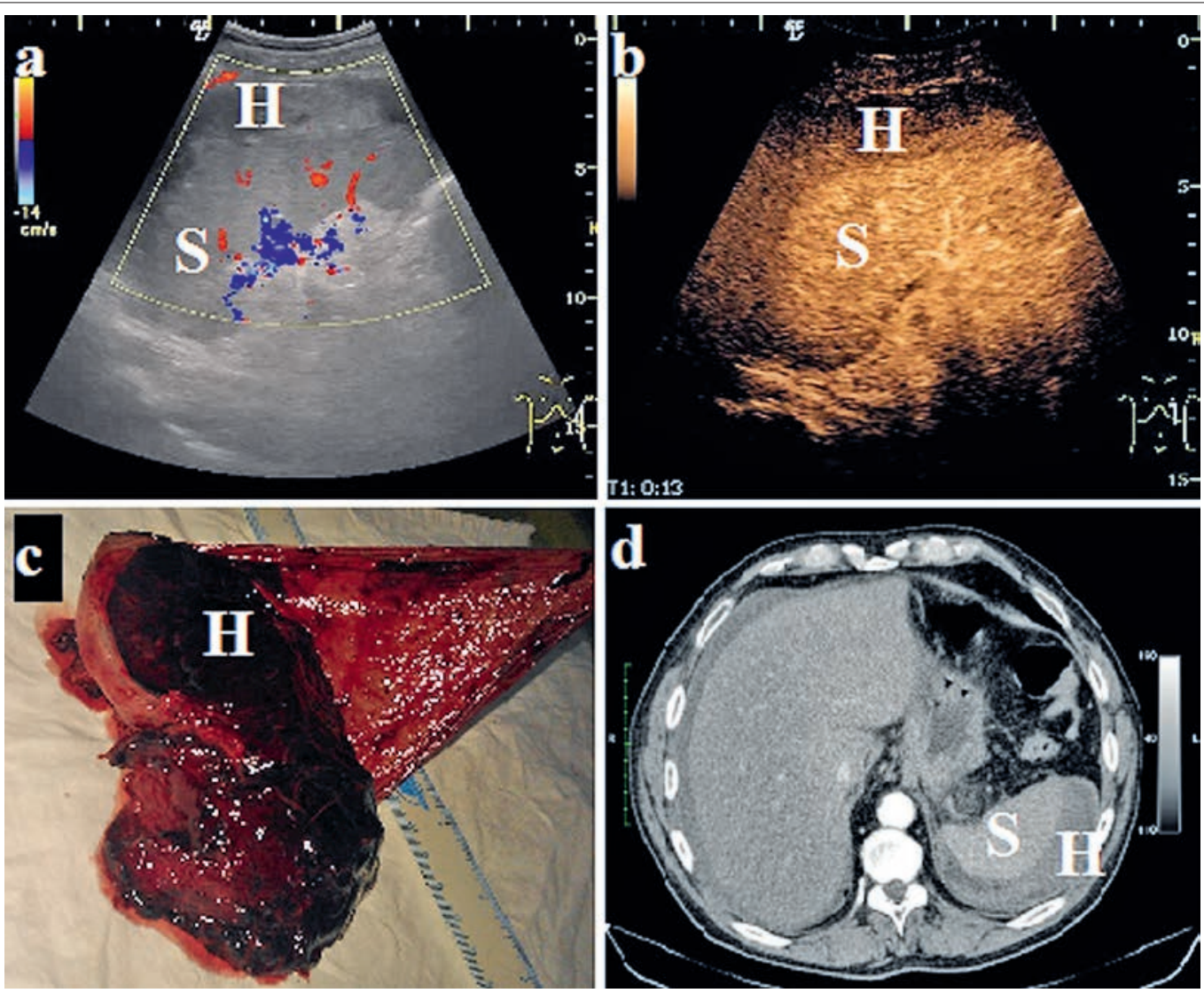

Fig 13. Large subcapsular hematoma. a) Absence of vascular Doppler signal inside hematoma; b) absence of contrast-agent extravasation inside hematoma, excluding active bleeding at examination time; c) surgically resected spleen - the large subcapsular hematoma is evident; d) abdominal contrast-enhanced CT scan, arterial time - subcapsular splenic hematoma, with inhomogeneous content, and perihepatic ascites $(\mathrm{S}=$ spleen, $\mathrm{H}=$ hematoma).

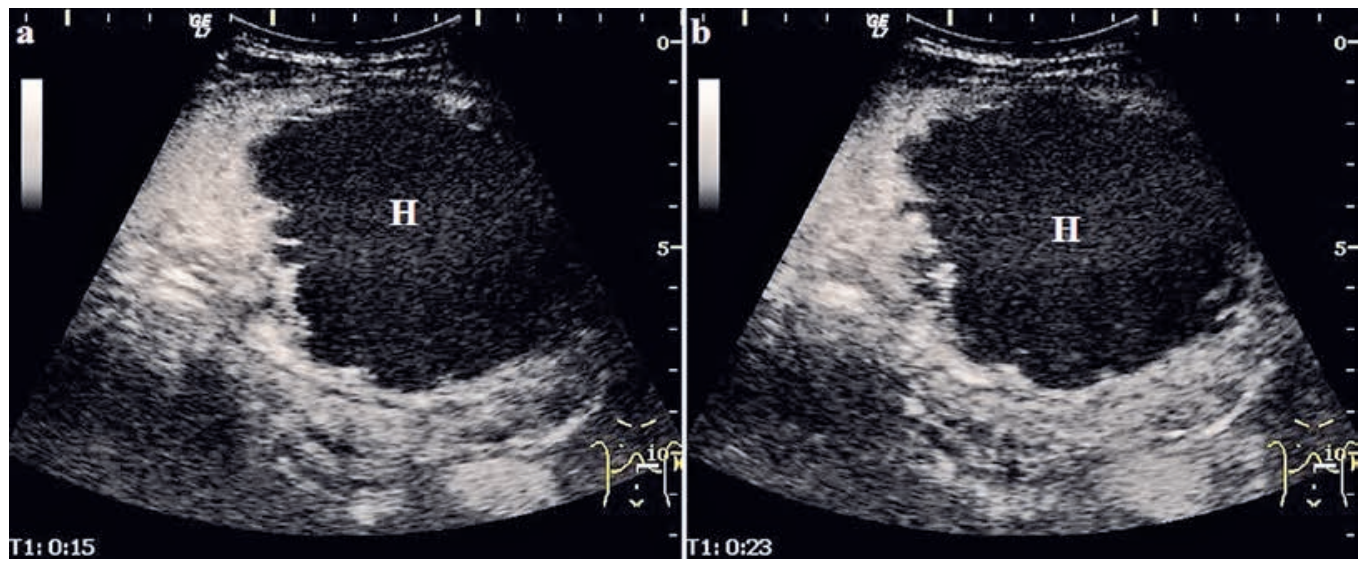

Fig 14. Old hematoma at CEUS. a) early arterial time - the hematoma has a clear content, without contrastagent inside; b) late arterial time - no extravasation of contrast inside the large hematoma $(\mathrm{H}=$ hematoma $)$. 

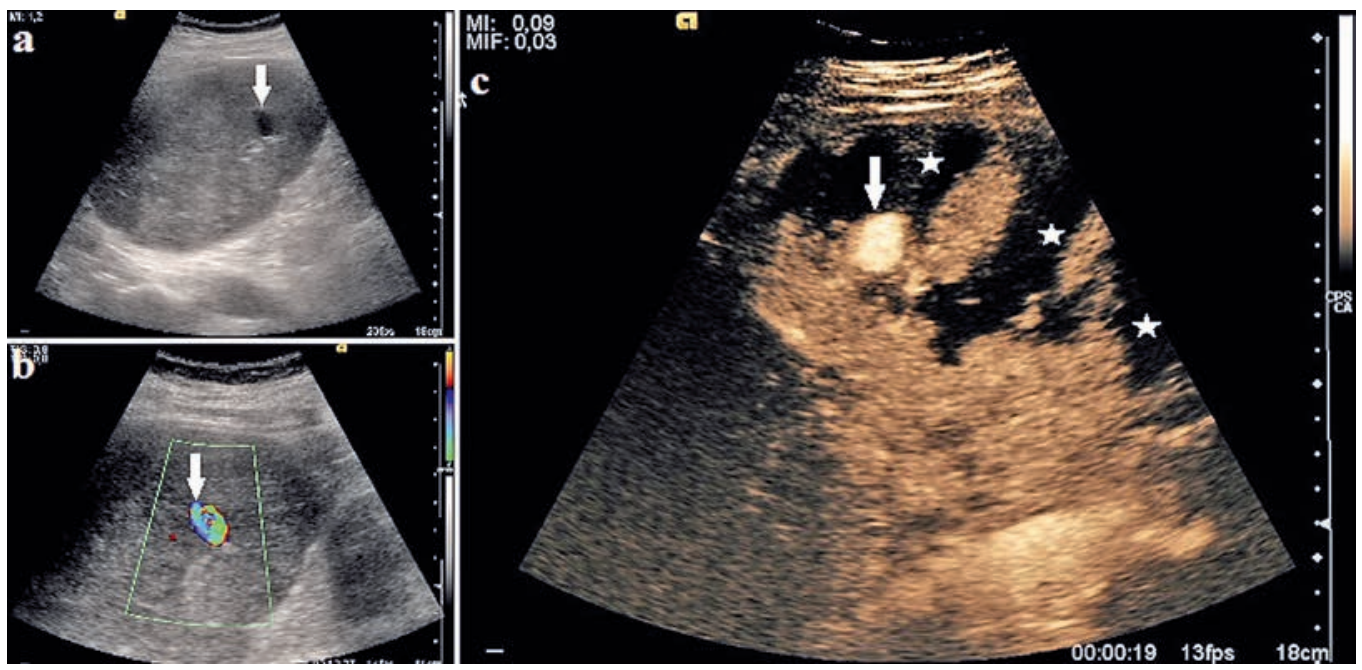

Fig 15. Non-Hodgkin infiltrative, diffuse lymphoma, with intratumoral fistula. a) sonogram shows an enlarged, globulous spleen, with diffuse inhommogenous structure, and an anechoic, well-delineated lesion (arrows); b) vascular signal inside the anechoic lesion at Doppler examination, pleading for an arterio-venous fistula; c) CEUS, arterial time (19 sec), inhomogeneous enhancement of the splenic parenchyma, with areas of low enhancement (asterisk), and the hyperenhanced vascular fistula can be well seen.
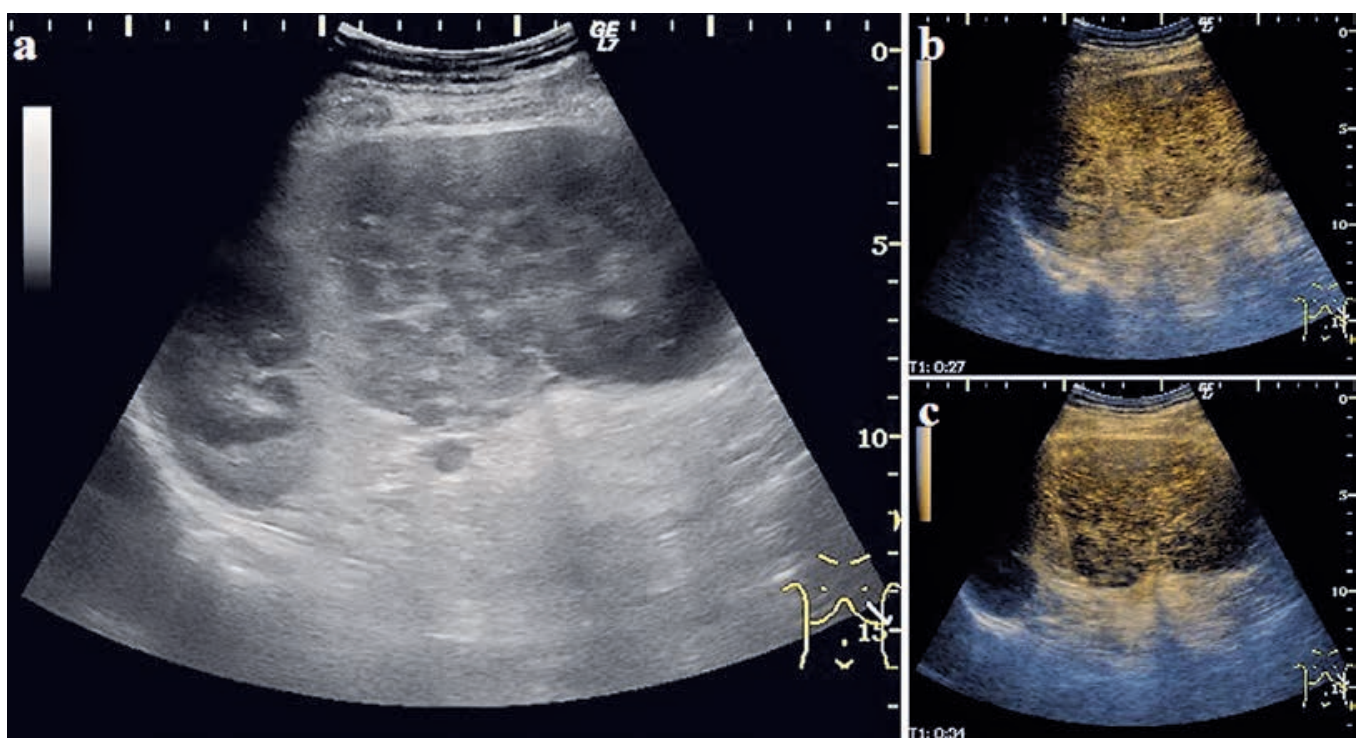

Fig 16. Non-Hodgkin miliary lymphoma. a) large and inhomogeneous spleen, with multiple micronodular hypoechoic lesions; b,c) CEUS, arterial and portal time - the micronodules show hypoenhancement in all phases.

dimension does not exclude the disease $[23,24]$. The lesions are hypoechoic in most of the cases, showing different US patterns: lesions smaller than $1 \mathrm{~cm}$ in diameter, with focal (miliary and nodular) or diffuse (infiltrative and diffuse) (fig 15, fig 16) destruction of splenic parenchyma (typical for non-Hodgkin lymphoma of low-grade malignancy), or focal lesions larger than $3 \mathrm{~cm}$, cyst-like (fig 17) (high-grade non-Hodgkin lymphoma). The indis- tinct boundary echo pattern is an important clue in differentiate them from cysts $[6,25]$. They are becoming more evident at CEUS as hypoechoic defects, less enhancing than the surrounding parenchyma, with lesion to parenchyma gradient progressively increasing while moving to the parenchymal phase. Especially in the diffuse form, it is difficult to be distinguished from hematogenous metastases. The lymphoma lesions are usually regularly 


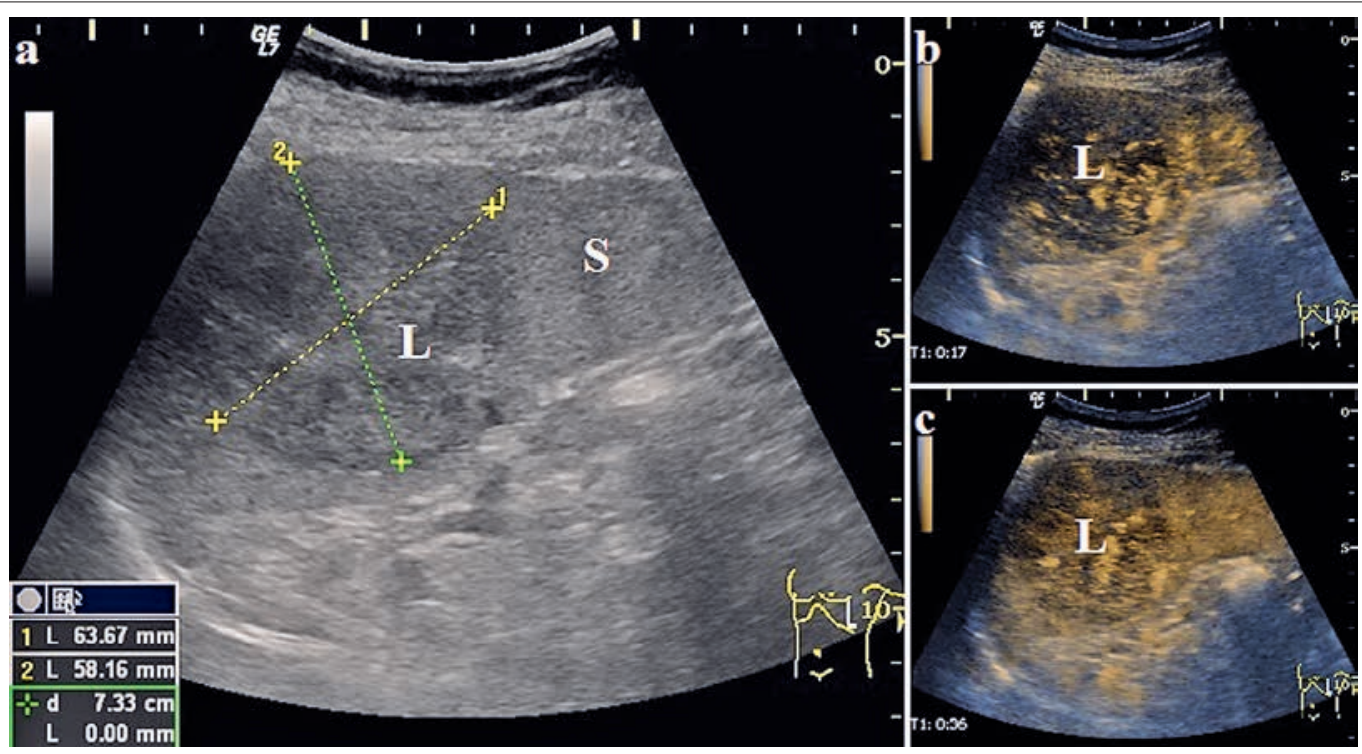

Fig 17. Non-Hodgkin cyst-like lymphoma. a) sonogram shows a splenic round, hypoechoic mass with indistinct boundaries; $b, c)$ the mass is hypoenhanced in all phases, being highly suggestive for malignancy $(\mathrm{S}=$ spleen, $\mathrm{L}=$ lymphoma).

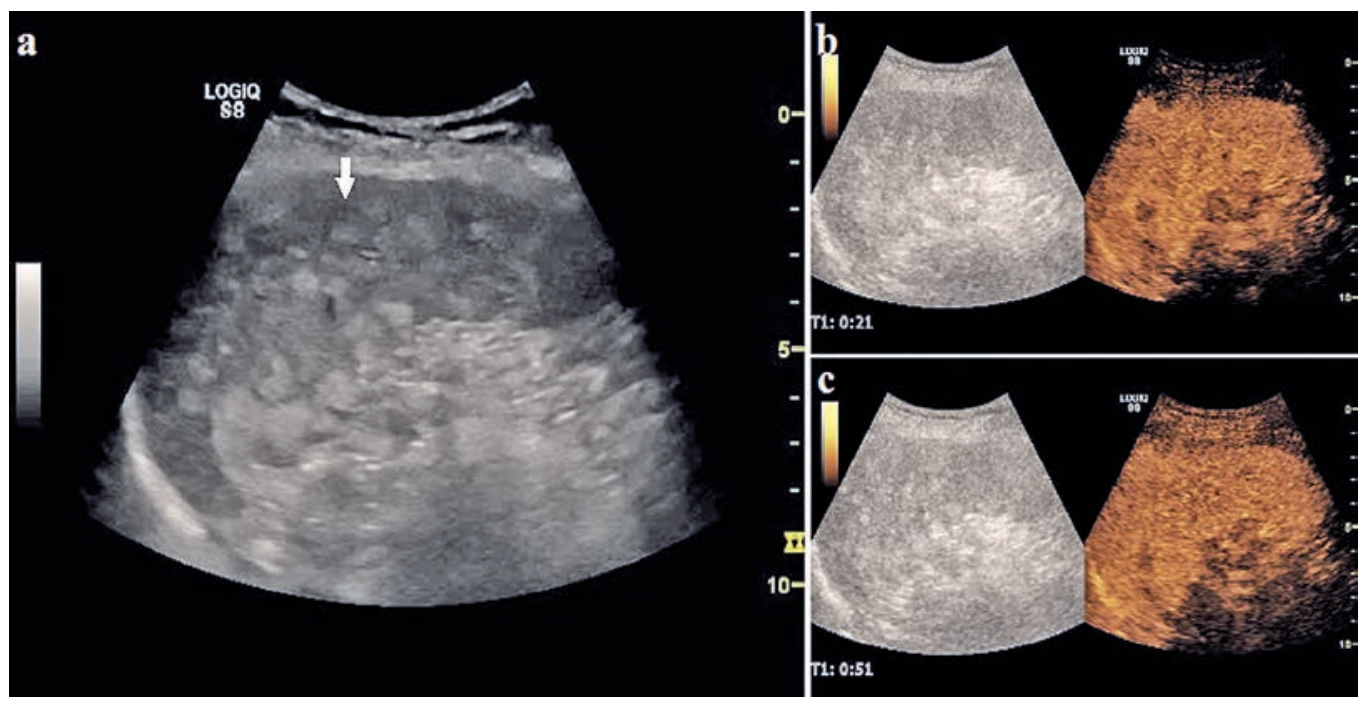

Fig 18. Multiple splenic metastasis from a colonic cancer. a) multiple hyperechoic splenic masses, anarchically disposed, some with hypoechoic rim (arrow); b,c) the lesions present an hypoenhancement pattern in all phases at CEUS.

deposited, while metastases are usually anarchically disposed $[2,13,26]$. Lesions with increased echogenicity are unusual in patients with lymphoma, and require histological confirmation $[2,13]$.

\section{Metastasis}

Splenic metastases are rare lesions, usually seen in patients with advanced malignant disease, having a poor prognosis. The most frequent metastases are from lym- phoma and melanoma, followed by carcinoma of the ovary, breast, lung, stomach, prostate, colon, liver, and pancreas $[5,8]$. As in malignant lymphoma, splenic metastases are predominantly hypoechoic, target lesions with a hypoechoic halo being suggestive for metastasis (sign of aggressive behavior) [13]. Sometimes hyperechoic (eg, carcinoma of the colon) or inhomogeneous lesions with a necrotic center can be found (rapid growth 

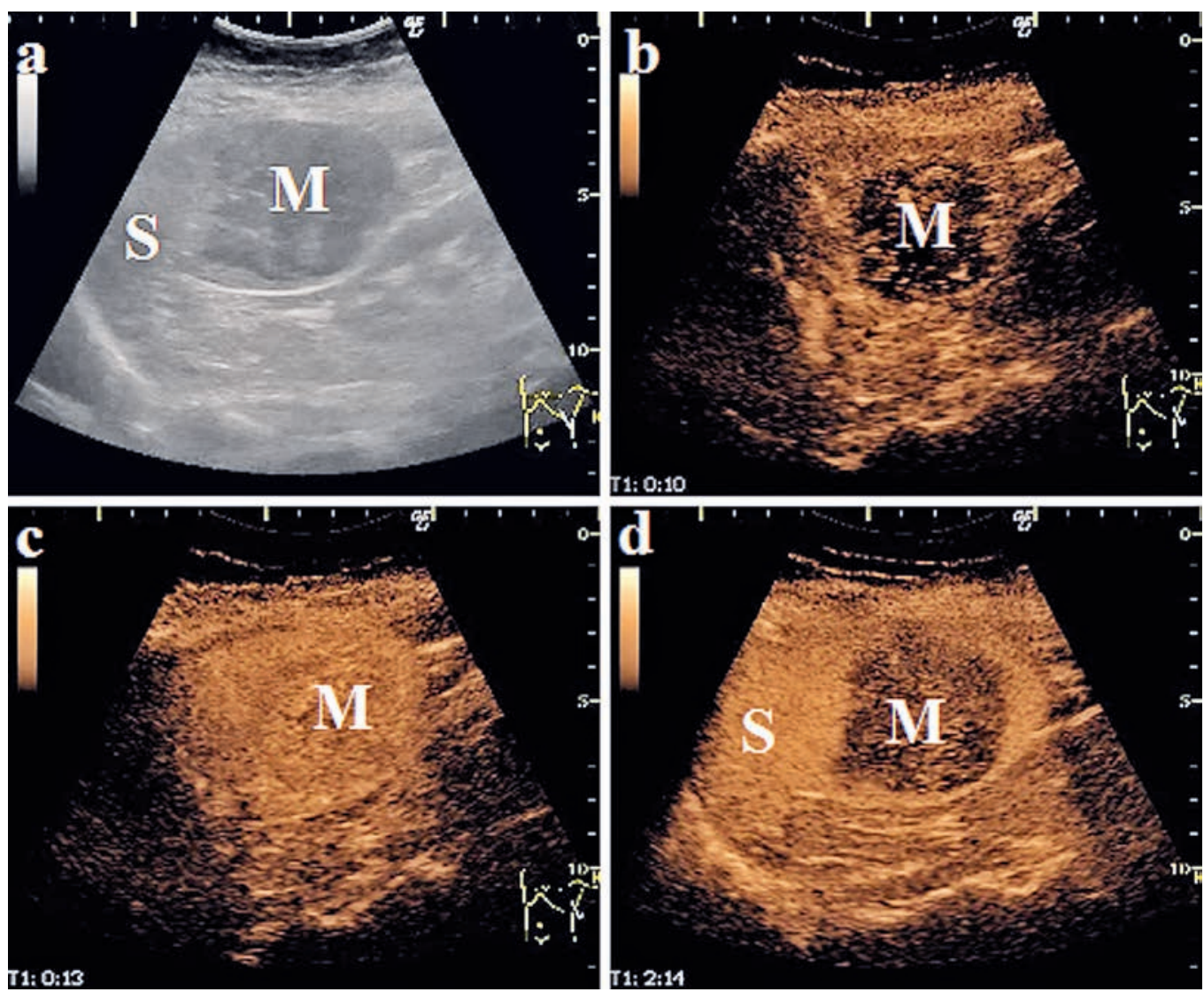

Fig 19. Hypoechoic, round splenic mass, highly suggestive for metastasis. a) 2D - large mass occupying the inferior pole of the spleen, with indistinct boundaries; b,c,d) CEUS showing a mass with early rapid and intense enhancement, and with wash-out $(\mathrm{M}=$ mass, $\mathrm{S}=$ spleen $)$.

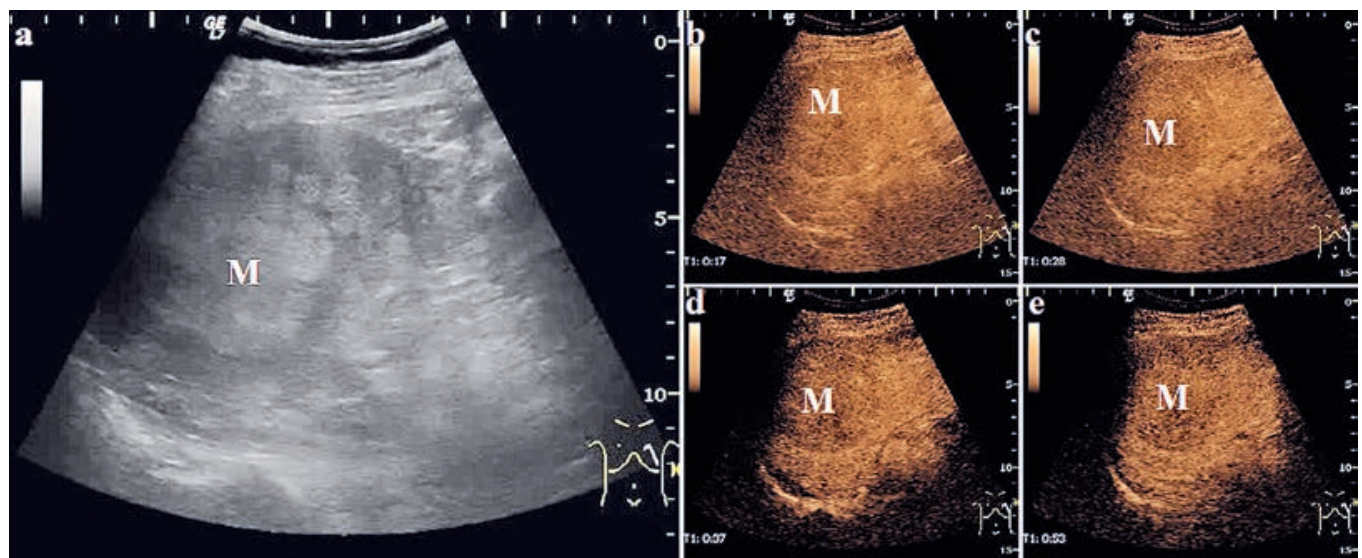

Fig 20. Splenic metastasis with unknown origin. a) gray-scale US showing a large, hyperechoic round splenic mass; b,c,d,e) CEUS - the lesion is hypoenhanced in all phases compared to the surrounding parenchyma $(\mathrm{M}=$ mass $)$. 
or mucinous origin - eg, ovarian carcinoma). With the exception of metastases of mucinous primary tumors, calcifications are rarely seen (fig 18-20) [27-29].

\section{Conclusion}

Sonography is a widely available, noninvasive, useful and valuable tool for diagnosing and follow-ups of splenic abnormalities, both benign and malignant. The additional use of CEUS can improve its diagnostic validity, especially when interpreted in the clinical context of the case. In many cases, pathologic confirmation is necessary to make a definitive diagnosis.

\section{References:}

1. Benter T, Klühs L, Teichgräber U. Sonography of the spleen. J Ultrasound Med 2011; 30: 1281-1293.

2. Catalano O, Sandomenico F, Matarazzo I, Siani A. Contrast-enhanced sonography of the spleen. AJR Am J Roentgenol 2005; 184: 1150-1156.

3. Von Herbay A, Barreiros AP, Ignee A, et al. Contrast-enhanced ultrasonography with SonoVue: differentiation between benign and malignant lesions of the spleen. J Ultrasound Med 2009; 28: 421-434.

4. Bamber J, Cosgrove D, Dietrich CF, et al. EFSUMB guidelines and recommendations on the clinical use of ultrasound elastography. Part 1: Basic principles and technology. U1traschall Med 2013; 34: 169-184.

5. Andrews MW. Ultrasound of the spleen. World J Surg 2000; 24: 183-187.

6. Chen MJ, Huang MJ, Chang WH, et al. Ultrasonography of splenic abnormalities. World J Gastroenterol 2005; 11: 4061-4066.

7. Bolondi L, Correas JM, Lencioni R, Weskott HP, Piscaglia F. New perspectives for the use of contrast-enhanced liver ultrasound in clinical practice. Dig Liver Dis 2007; 39: 187195.

8. Wan YL, Cheung YC, Lui KW, Tseng JH, Lee TY. U1trasonographic findings and differentiation of benign and malignant focal splenic lesions. Postgrad Med J 2000; 76: 488-493.

9. Stang A, Keles H, Hentschke S, et al. Differentiation of benign from malignant focal splenic lesions using sulphur hexafluoride-filled microbubble contrast-enhanced pulseinversion sonography. AJR Am J Roengenol 2009; 193: 709-721.

10. Catalano O, Lobianco R, Sandomenico F, D’Elia G, Siani A. Real-time contrast-enhanced ultrasound of the spleen: examination technique and preliminary clinical experience. Radiol Med 2003; 106: 338-356.

11. Catalano O, Sandomenico F, Vallone P, D'Errico A, Siani A. Contrast-enhanced sonography of the spleen. Semin U1trasound CT MR 2006; 27: 426-433.
12. Tafuto S, Catalano O, Barba G, et al. Real-time contrastenhanced specific ultrasound in staging and follow-up of splenic lymphomas. Front Biosci 2006; 11: 2224-2229.

13. Goerg C, Schwerk WB, Goerg K. Sonography of focal lesions of the spleen. AJR Am J Roentgenol 1991; 156: 949953.

14. Balzan SM, Riedner CE, Santos LM, Pazzinatto MC, Fontes PR. Posttraumatic splenic cysts and partial splenectomy: report of a case. Surg Today 2001; 31: 262-265.

15. Giovagnoni A, Giorgi C, Goteri C. Tumors of the spleen. Cancer Imaging 2005; 5: 73-77.

16. Caremani M, Occhini U, Caremani A, et al. Focal splenic lesions: US findings. J Ultrasound 2013; 16: 65-74.

17. Lim AK, Patel N, Eckersley RJ, Taylor-Rosbinson SD, Cosgrove DO, Blomley MJ. Evidence for spleen-specific uptake of a microbubble contrast agent: a quantitative study in healthy volunteers. Radiology 2004; 231: 785-788.

18. Gorg C, Bert T. Second-generation sonographic contrast agent for differential diagnosis of perisplenic lesions. AJR Am J Roentgenol 2007; 186: 621-626.

19. Changchien CS, Tsai TL, Hu TH, Chiou SS, Kuo CH. Sonographic patterns of splenic abscess: an analysis of 34 proven cases. Abdom Imaging 2002; 27: 739-745.

20. McKenney KL, Nuñez DB Jr, McKenney MG, Asher J, Zelnick K, Shipshak D. Sonography as the primary screening technique for blunt abdominal trauma: experience with 899 patients. AJR Am J Roentgenol 1998; 170: 979-985.

21. Catalano O, Lobianco R, Sandomenico F, Siani A. Splenic trauma: evaluation with contrast-specific sonography and a second-generation contrast medium - preliminary experience. J Ultrasound Med 2003; 22: 467-477.

22. Goerg C, Colle J, Goerg K, Prinz H, Zugmaier G. Spontaneous rupture of the spleen: ultrasound patterns, diagnosis and follow-up. Br J Radiol 2003; 76: 704-711.

23. Goerg C, Schwerk WB, Goerg K, Havemann K. Sonographic patterns of the affected spleen in malignant lymphoma. J Clin Ultrasound 1990; 18: 569-574.

24. Abbott RM, Levy AD, Aguilera NS, Gorospe L, Thompson WM. From the archives of the AFIP: primary vascular neoplasms of the spleen - radiologic-pathologic correlation. Radiographics 2004; 24: 1137-1163.

25. Urrutia M, Mergo PJ, Ros LH, Torres GM, Ros PR. Cystic masses of the spleen: radiologic-pathologic correlation. Radiographics 1996; 16: 107-129.

26. Robertson F, Leander P, Ekberg O. Radiology of the spleen. Eur Radiol 2001; 11: 80-95.

27. Alloni R, Garberini A, Caputo D, Coppola R. Solitary splenic metastasis of ovarian carcinoma: report of two cases. Surg Today 2008; 38: 1144-1147.

28. Compérat E, Bardier-Dupas A, Camparo P, Capron F, Charlotte F. Splenic metastases: clinicopathologic presentation, differential diagnosis, and pathogenesis. Arch Pathol Lab Med 2007; 131: 965-969.

29. Ushijima K, Nishida T, Okura N, et al. Solitary splenic recurrence of ovarian cancer: case report and review of the literature. Arch Gynecol Obstet 1999; 263: 79-81. 